\title{
Synthesis and Characterization of Polysulfone (PSU)/Philippine Halloysite (PH-HAL) Nanostructured Membrane via Electrospinning
}

\author{
Ruth R. Aquino ${ }^{1}$, Marvin S. Tolentino ${ }^{1}$, Niel Karl G. Arcamo ${ }^{1}$, John Patrick N. Gara ${ }^{1}$, and Blessie A. Basilia ${ }^{1,2}$ \\ ${ }^{1}$ Mapúa University, School of Chemical, Biological, and Materials Engineering and Sciences, 658 Muralla St., Intramuros Manila, 1002 \\ Philippines \\ ${ }^{2}$ Industrial Technology Development Institute, Department of Science and Technology, Bicutan, Taguig City, Metro Manila, 1631Philippines
}

\begin{abstract}
Membrane technology is widely used in many separation processes because of its multi-disciplinary characteristics. One of the techniques that is used in the fabrication of membranes is the electrospinning process which can create nanofibers from a very wide range of polymeric materials. In this study, electrospun nanostructured fibrous composite membranes of polysulfone (PSU), commercial halloysite (COM-HAL), and Philippine halloysite (PH-HAL) were synthesized. The concentrations of COM-HAL and PH-HAL were both varied from $0.5 \%, 1 \%$, and $2 \%$. The FTIR results showed that there were changes in the intensity of the PSU-IR spectra which confirmed the presence of COM-HAL and PH-HAL in the synthesized membranes. The SEM revealed that nanofibers can be successfully produced by the addition of $\mathrm{LiCl}$ salt in PSU with varying $\mathrm{HAL}$ concentrations. Also, it was observed that the addition of HAL with varying concentrations have no significant effect on wettability due to the strong hydrophobic character of the PSU membrane. Moreover, it was found from the analysis of mechanical properties that the tensile strength of the membranes weakened by the addition of HAL due to its weak interaction with PSU.
\end{abstract}

\section{Introduction}

Membrane technology is an emerging technology which can be used in many separation processes. Numerous membrane processes emerged in which applications are based on different separation principles and mechanisms. Various techniques and fabrication methods have been explored to synthesize membranes, and one of those techniques is the electrospinning process.

Electrospinning is a versatile process that can create nanofibers from a very wide range of polymeric materials. The practicality of electrospinning has been greatly improved with recent advances in mass production scalability, leading to higher production rate and lower cost materials. Moreover, the addition of salt concentration to polymer solution improves the nanofiber formation of the membrane [1]. One of the widely-used polymer materials for electrospinning is polysulfone (PSU). Since it exhibits excellent thermal, mechanical, chemical stability, and low cost, PSU is widely used in separation processes such as water and wastewater treatment, chemical, metallurgical, and bioseparation area. Previous research studied the influence of addition of nanoclay such as halloysite (HAL) on PSU nanocomposite to serve as excellent support for nanoparticles due to their unique geometry and properties. However, most of the work done on the addition of clay to PSU nanocomposite membranes had been prepared using dispersion methods.

This study focused on the synthesis and characterization of a nanostructured PSU/HAL membranes via electrospinning process. Specifically, this study aims to: (1) determine the effect of $\mathrm{LiCl}$ on the surface morphology of the nanostructured membrane; and (2) compare the following mixtures of electrospun membranes: pure PSU, PSU/LiCl, PSU/LiCl with commercial halloysite (PSU/LiCL/COM-HAL), and $\mathrm{PSU} / \mathrm{LiCl}$ with Philippine halloysite (PSU/LiCl/PH-HAL) using different characterization techniques; namely, (a) effect of HAL addition on the chemical composition of the membrane by Fourier Transform Infrared (FTIR) analysis, (b) surface morphology through Scanning Electron Microscopy (SEM), (c) wettability of the surface with the use of contact angle goniometer, and (c) mechanical properties using the Universal Testing Machine.

Considering the low cost and availability of PSU and HAL, this study would serve as guide on the proper and efficient way of fabricating PSU incorporated with HAL. Also, this undertaking may serve as a gateway for further improvements and use of $\mathrm{PSU} / \mathrm{LiCl} / \mathrm{HAL}$ nanofiber membrane via electrospinning into various areas of applications.

\section{Methodology}

PSF, with an average molecular weight of 35,000 , and COM-HAL were both obtained from Sigma-Aldrich. Lithium chloride $(\mathrm{LiCl})$ salt and anhydrous dimethylacetamide $99.8 \%$ (DMAc) were obtained from the Mapúa University Chemical Supplies. PH-HAL was 
obtained from the Department of Science and Technology of the Philippines. The electrospinning procedure was partly based on methodology of Chang and Lin (2009) [1]. PSU mixtures were prepared at different compositions; specifically, pure PSU, PSU/LiCl, $\mathrm{PSU} / \mathrm{LiCl}$ with varying concentrations of COM-HAL $(0.5 \%, 1 \%$ and $2 \%)$, and $\mathrm{PSU} / \mathrm{LiCl}$ with varying concentrations of PH-HAL $(0.5 \%, 1 \%$ and $2 \%) .40 \mathrm{~g}$ of each polymer solution was prepared and stirred at $60{ }^{\circ} \mathrm{C}$ for $6 \mathrm{~h}$. Initially, the solvent was prepared in a media bottle and preheated by submerging it in a water bath at $60{ }^{\circ} \mathrm{C}$ for 15 mins prior to dissolving the solutes. The flow rate of the solution and the voltage was set to 1.0 $\mathrm{mL} \mathrm{h-1}$ and $25 \mathrm{kV}$, respectively. The tip-to-collector

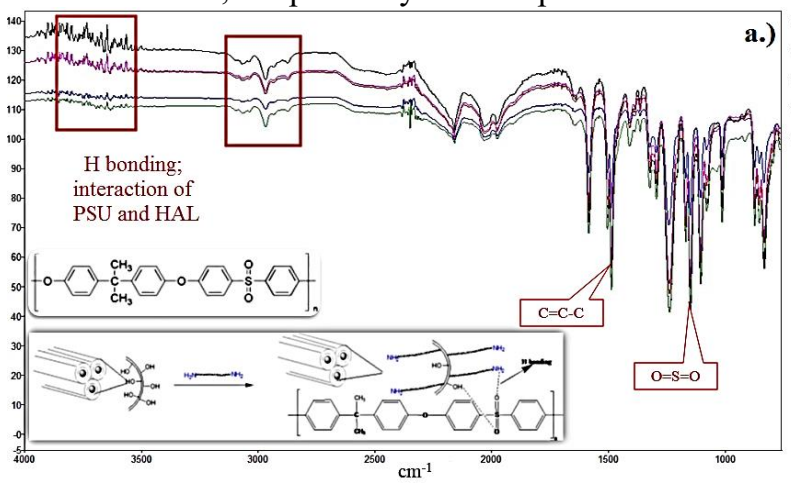

Figure 1.a.) IR spectrum of thesynthesized PSU/COM-HAL me

\section{Results and discussion}

\subsection{Effect of HAL on chemical composition of the membranes}

Based on the results of FTIR analysis (Figure 1.a.), the IR spectra of PSU and PSU/COM-HAL at different concentrations of COM-HAL were observed. For pure PSU, band peaks of the different functional groups present were determined having the following wavenumbers: $1013 \mathrm{~cm}^{-1}$ and $1103 \mathrm{~cm}^{-1}$ (aromatic $\mathrm{C}-\mathrm{H}$ in-plane bend); $1148 \mathrm{~cm}^{-1}$ and $1171 \mathrm{~cm}^{-1}(\mathrm{O}=\mathrm{S}=\mathrm{O}$ symmetrical stretch); $1239 \mathrm{~cm}^{-1}(\mathrm{C}-\mathrm{O}-\mathrm{C}$ symmetrical stretch); $1292 \mathrm{~cm}^{-1}$ and $1322 \mathrm{~cm}^{-1}(\mathrm{O}=\mathrm{S}=\mathrm{O}$ symmetrical stretch); $1488 \mathrm{~cm}^{-1}$ and $1586 \mathrm{~cm}^{-1}(\mathrm{C}=\mathrm{C}-\mathrm{C}$ aromatic ring stretch); $2966 \quad \mathrm{~cm}^{-1} \quad$ (methyl $\mathrm{C}-\mathrm{H}$ asymmetrical/symmetrical stretch). The band peaks in the IR spectra of PSU were all evident which confirmed the presence of PSU in the membrane. Moreover, the HAL IR spectra were also observed in accordance to the FTIR analysis by Bordeepong et al. (2011) [2]. The similarity between the obtained IR spectra for PSU/COM-HAL (Figure 1.a) and the expected IR spectrum for PSU/HAL (Figure 1.b) confirmed the interaction between PSU and HAL.

\subsection{Effect of $\mathrm{LiCl}$ and HAL to the membrane surface morphology}

Figure 2.b illustrates the SEM image of the electrospun PSU with the addition of $\mathrm{LiCl}$, and as observed smooth distance was set to $15 \mathrm{~cm}$. The equipment used to detect the chemical components and functional groups of the membranes was PerkinElmer Spectrum 100 FT-IR Spectrometer. The electrospun membranes were subjected to SEC Mini-SEM SNE-3200M micrograph analysis with $10 \mathrm{kV}$ accelerating voltage for the comparison of surface morphologies. Moreover, 25 fiber diameters were measured using ImageJ ver. $1.50 \mathrm{i}$ software to calculate the average fiber diameter. Also, contact angle goniometer was used to determine the wettability of the membranes. The mechanical properties of the membranes were determined using Instron 3225 Single Column UTM.

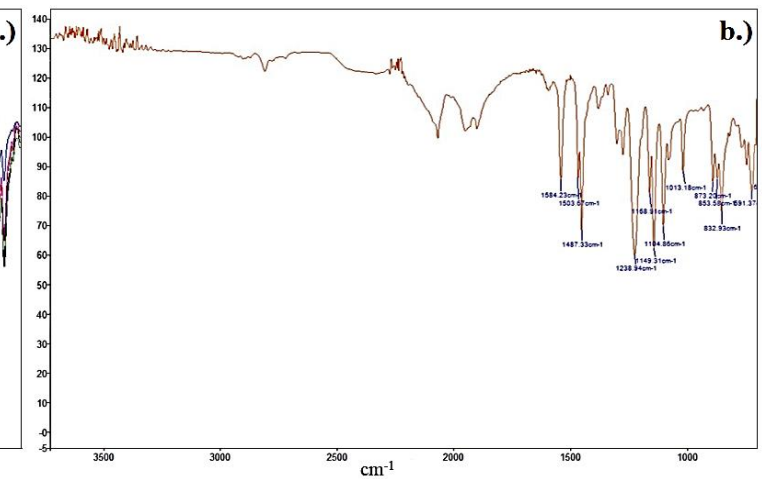

embranesandb.) expected PSU/HAL membrane IRspectrum.

fibers without beads and uniform fibers were produced as compared with pure PSU (Figure 2.a).

The obtained results confirmed that the addition of salt improved the conductivity of polymer solution which helps fiber formation significantly [1], but as observed, it also resulted to the decrease in the average fiber diameter from $697.37 \pm 193.20 \mathrm{~nm}$ for pure PSU to $372.72 \pm$ $130.50 \mathrm{~nm}$ for PSU/LiCl (Figure 3).

With regards to the addition of $\mathrm{HAL}$ to $\mathrm{PSU} / \mathrm{LiCl}$, curvy fibers with slight spindle-like bead can be observed with $0.5 \%$ COM-HAL (Figure 2.c) with an average fiber diameter of $521.78 \pm 166.14 \mathrm{~nm}$. Further increase in COM-HAL concentration ( $1 \%$ and $2 \%)$ showed no significant changes in the surface morphologies (Figures 2.d and 2.e), but it can be seen that the average fiber diameter somehow increases (Figure 3). For the addition of $\mathrm{PH}-\mathrm{HAL}$ to $\mathrm{PSU} / \mathrm{LiCl}, 0.5 \%$ and $1 \%$ concentrations resulted to the formation of spindle-like bead and beadon-string morphologies (Figures 2.f and 2.g) with an average fiber diameter of $403.38 \pm 99.000 \mathrm{~nm}$. It can be seen in Figure 3, an increasing trend can also be observed for the average fiber diameter as the concentration of $\mathrm{PH}$ HAL increases.

The SEM micrographs revealed that the average fiber diameter decreased significantly with the addition of $\mathrm{LiCl}$ to PSU membrane. Conversely, the incorporation of COM-HAL and PH-HAL on the membrane increased the average fiber diameter.

\subsection{Effect of HAL to the wettability of the membrane}




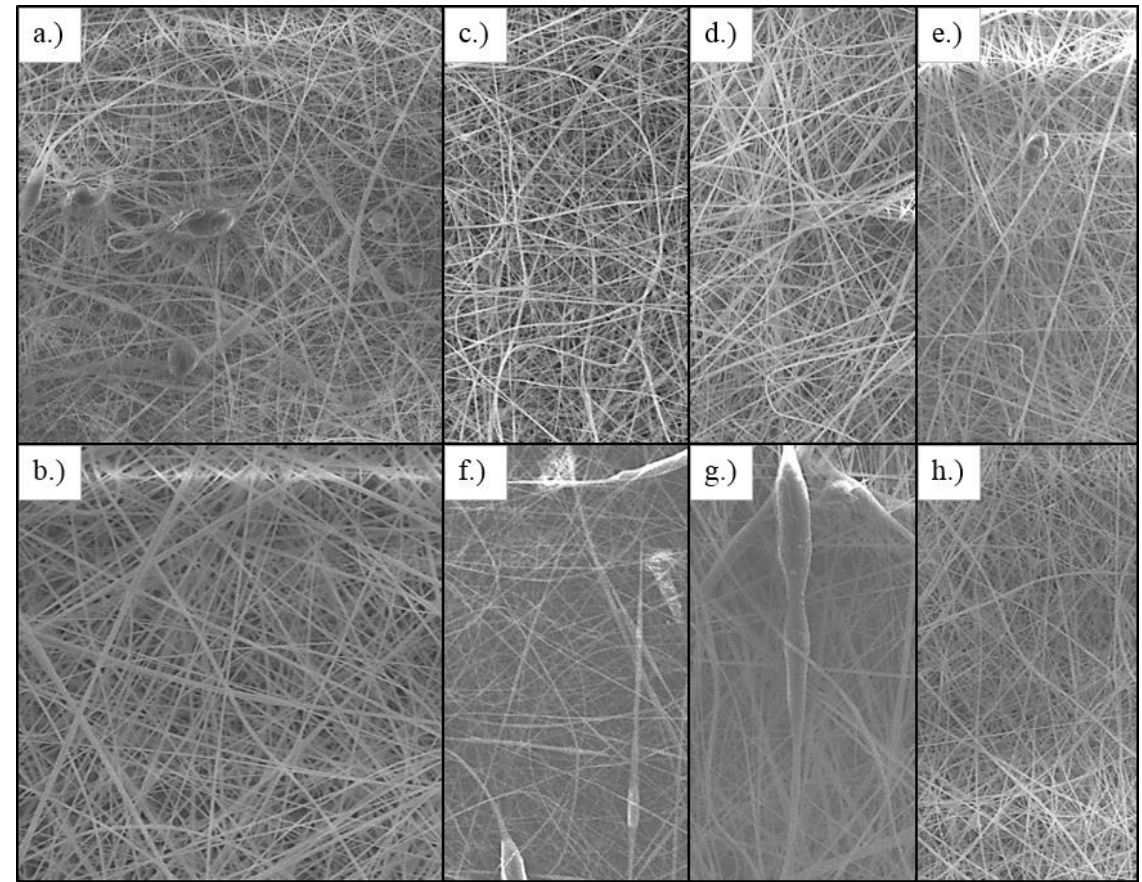

Figure 2. SEM micrographs of the electrospun membranes: a.) pure PSU; b.) PSU/LiCl; PSU/LiCl/COM-HAL at c.) $0.5 \%$, d.) $1 \%$, and e.) $2 \%$ COM-HAL concentrations; and PSU/LiCl/PH-HAL at f.) $0.5 \%$, g.) $1 \%$, and h.) $2 \%$ PH-HAL concentrations.

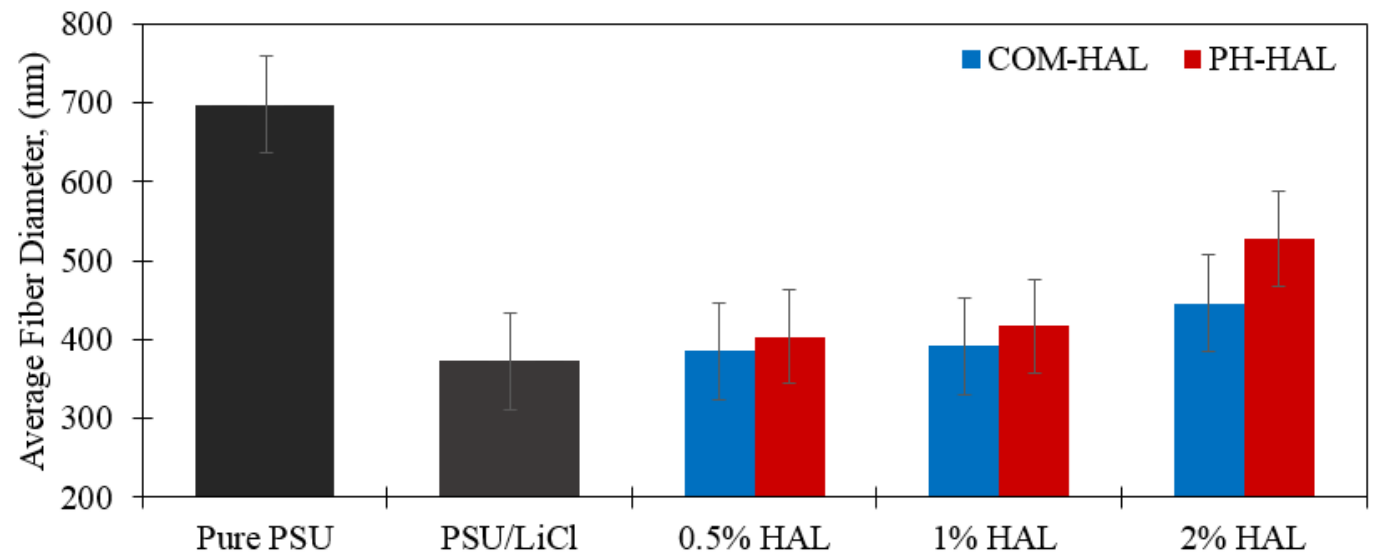

Figure 3. Effect of $\mathrm{LiCl}$ and $\mathrm{HAL}$ to the average fiber diameter of the membranes.

The contact angle of water on pure PSU was approximately $142 \pm 2.94^{\circ}$ which is in good agreement with the strong hydrophobic nature of PSU. Based on the contact angle measurements of water on the electrospun membranes, the addition of $\mathrm{LiCl}$ and HAL had no significant effect on the wettability of the membranes (Figure 4). The statistical analysis that was conducted, wherein p-value was greater than the significance level, also showed that hydrophobicity of the composite membranes was not affected by the incorporation of HAL. Although HAL is hydrophilic in nature, the increasing HAL content caused the surface of the nanofiber membranes to become rougher, which led to the decrease of the interfacial tension and surface energy [3]. Thus, the contact angle measurements lie within the range of each other.

\subsection{Effect of HAL to the mechanical properties of the membranes}

As shown in Figure 5.a, increasing the concentration of PH-HAL decreases the tensile strength of the composite membrane. This is due to the weak interaction between PSU and HAL. The phenomenon was observed in the study of Peng et al. (2009) [4] in which they stated that the addition of nanoclay to the nanocomposites loss of tensile strength is due to the decreasing uniaxial orientation. Similar effect can be also observed with the addition of COM-HAL. Although there is a sudden increase in the tensile strength at $1 \%$ COM-HAL, the statistical analysis resulted to a p-value greater than the significance level suggesting that the addition of COMHAL and PH-HAL had no significant effect to the tensile strength of the membranes. Moreover, it can be seen on the graph (Figure 5.b) that the addition of PH-HAL increased the maximum strain of the membrane as compared to the effect of adding COM-HAL. For the modulus (Figure 5.c), the incorporation of $\mathrm{LiCl}$ had no effect, but the values decreases with the addition of both COM-HAL and PH-HAL $(0.5 \%$ and $2 \%)$. A sudden 
increase that can be observed at $1 \%$ concentration was due to the poor dispersion of the clay to the composite films resulting to high modulus. Considering the maximum tensile extension (Figure 5.d), the values increases with the concentration of COM-HAL, but no specific trend can be seen with the addition of PH-HAL. In general, decrease in the mechanical properties can be observed due to the effect of increasing HAL content

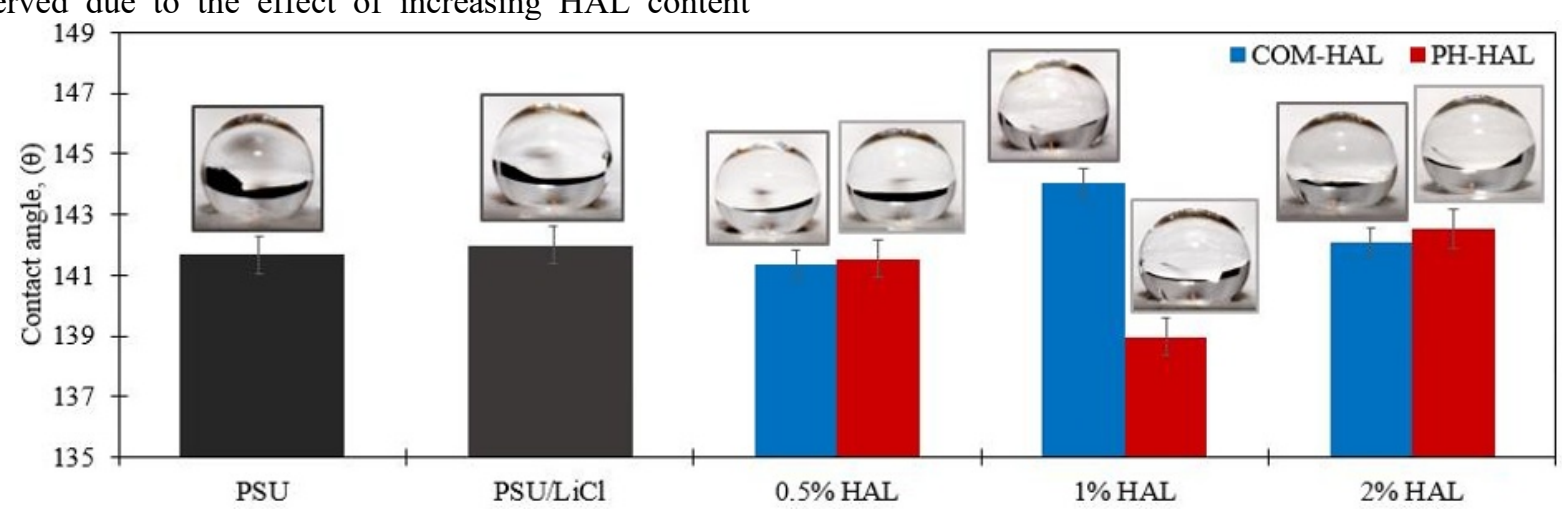

Figure 4. Contact angle measurements for the fabricated membranes
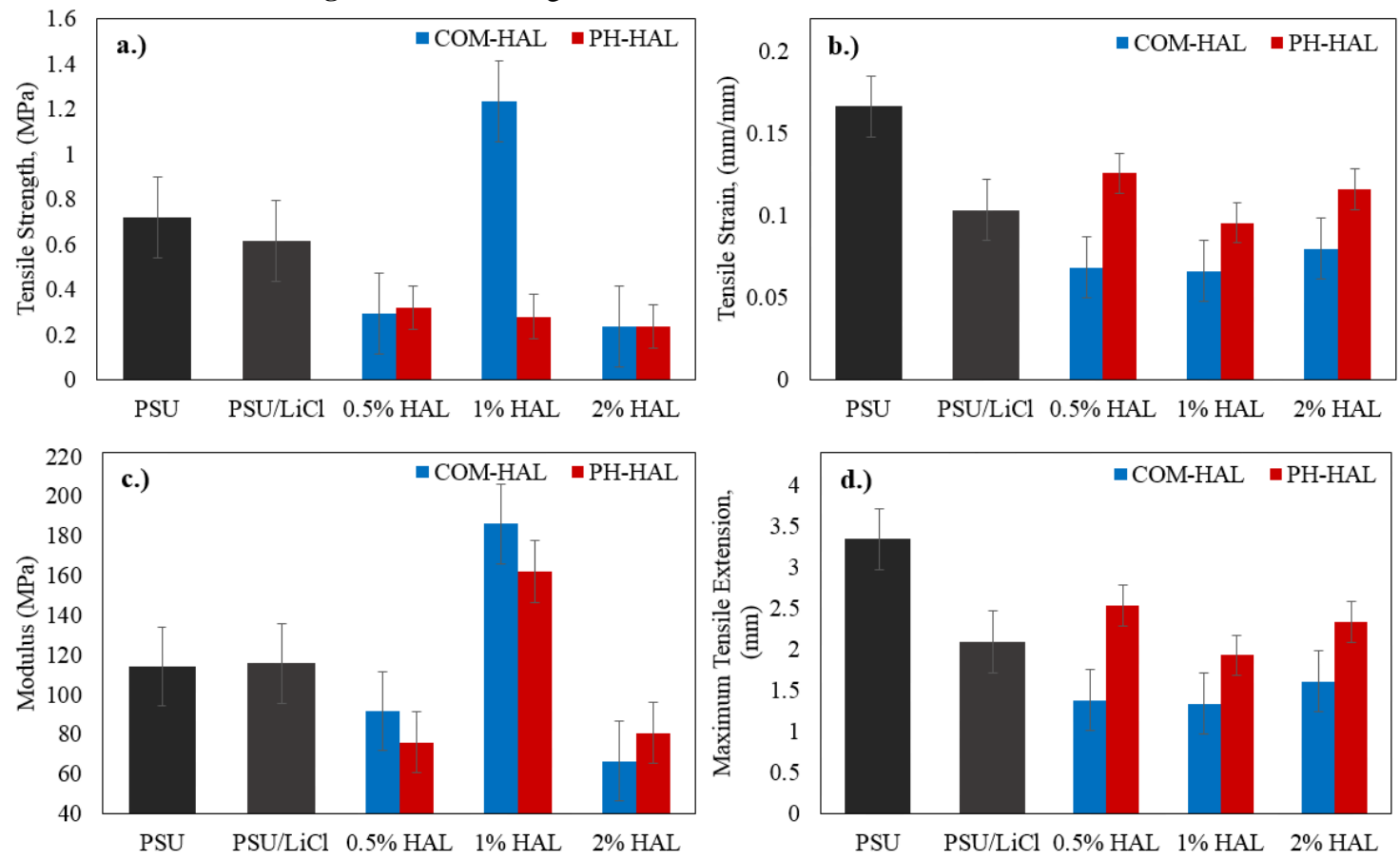

Figure 5. Mechanical properties of the electrospun membranes: a.) Tensile Strength, b.) Tensile Strain, c.) Modulus, and d.) Maximum Tensile Extension.

\section{Conclusion}

The researchers were able to synthesize nanofibrous membranes of PSU which contains COM-HAL and PHHAL by electrospinning technique. It was observed form the SEM micrograph that the addition of $\mathrm{LiCl}$ salt produced bead free nanofibers with reduced average fiber diameters. The FTIR analysis verified the hydrogen bonding interaction of PSU and HAL with changing band peaks intensities from $3600 \mathrm{~cm}^{-1}$ to $3900 \mathrm{~cm}^{-1}$. The addition of HAL on the membrane had no significant effect on the wettability of PSU. The composite which weakened the membrane; thus, decreasing the tensile strength [5]. However, upon statistical treatment of the data gathered, the p-values were found to be large and greater than the significance level which suggest that the addition of COM-HAL and PH-HAL at the concentrations used had no significant effect on the PSU membrane. 


\section{References}

1. K. Chang and H. Lin, Electrospin of polysulfone in N,N'-dimethylacetamide solutions. Journal of Polymer Research, 16, 611-622 (2009).

2. S. Bordeepong, D. Bhongsuwan, T. Pungrassami, and T. Bhongsuwan, Characterization of halloysite from thungyai district, Nakhon $\mathrm{Si}$ Thammarat Province, in Southern Thailand. Songklanakarin. $J$. Sci. Technol., 33, 599-607 (2011).

3. C. Luo, Z. Zou, B. Luo, W. Wen, H. Li, and M. Liu, Enhanced mechanical properties and cytocompatibility of electrospunpoly(L-lactide) composite fiber membranes assisted bypolydopamine-coated halloysite nanotubes, Applied Surface Science, 369, 82-91 (2016).

4. Q. Peng, P. Cong, X. Liu, T. Liu, S. Huang and T. $\mathrm{Li}$, The preparation of PVDF/clay nanocomposites and the investigation of their tribologicalproperties, Wear, 266(7-8), 713-720 (2009).

5. Y. Ma, F. Shi, Z. Wang, M. Wu, J. Ma and C. Gao, Preparation and characterization of PSf/clay nanocomposite membranes with PEG 400 as a pore forming additive. Desalination, 286, 131- 137 (2012). 